\title{
The role of dietary gangliosides on immunity and the prevention of infection
}

\author{
Ricardo Rueda* \\ Dept. of Science and Technology, Abbott Nutrition, Granada, Spain
}

Gangliosides are acid glycosphingolipids widely distributed in most vertebrate tissues and fluids. They are present in mammalian milk, where they are almost exclusively associated with the membrane fraction of the fat globule. In human milk, the content and individual distribution of gangliosides changes during lactation, $\mathrm{GD}_{3}$ being the most abundant ganglioside in colostrum, while in mature milk, $\mathrm{GM}_{3}$ is the major individual species. Gangliosides function as "unintended" target receptors for bacterial adhesion in specific tissues. After oral administration, they can be putative decoys that interfere with pathogenic binding in the intestine, this being the main mechanism by which these compounds can prevent infection. Ganglioside-supplemented infant formula has been reported to modify the intestinal ecology of preterm newborns, increasing the Bifidobacteria content and lowering that of Escherichia coli. In addition, the influence of dietary gangliosides on several parameters related to the development of intestinal immune system, such as cytokine and intestinal IgA production, has also been described in animal models. Recently, the influence of $\mathrm{GM}_{3}$ and $\mathrm{GD}_{3}$ on dendritic cell maturation and effector functionalities has also been reported, suggesting a role for these milk gangliosides, especially $\mathrm{GD}_{3}$, in modulating the process of oral tolerance during first stages of life. In summary, dietary gangliosides may have an important role in the modification of intestinal microflora and the promotion of intestinal immunity development in the neonate, and consequently in the prevention of infections during early infancy.

\section{Human milk: Gangliosides: Infection: Immunity}

Gangliosides are negatively charged glycosphingolipids that consist of a hydrophobic ceramide and a hydrophilic oligosaccharide chain bearing one or more sialic acid residues in addition to a number of sugars, namely glucose, galactose, $\mathrm{N}$-acetylglucosamine and $\mathrm{N}$-acetylgalactosamine ${ }^{1}$. Ceramide is an N-acylsphingosine in which the acyl residue is usually a saturated fatty acid with a chain length of more than 14 carbons. Svennerholm developed the classical and most frequently used nomenclature for gangliosides, which is based on two letters and one subscript number ${ }^{2}$. The first letter indicates the "series", which is different depending on the carbohydrate core and metabolic pathway (for example, $\mathrm{G}=$ ganglio series). The second letter indicates the number of sialic acid residues $(\mathrm{M}, \mathrm{D}, \mathrm{T}, \mathrm{Q}, \mathrm{P}, \mathrm{H}$ or $\mathrm{S}$, corresponding to one, two, three, four, five, or exceptionally, six or seven residues). Finally, the subscript corresponds to five minus the number of neutral monosaccharides residues present in the molecule. Attempts to develop more systematic approaches to naming gangliosides, such as that established by the Commission of the International Union of Biochemistry ${ }^{1}$ have not gained popularity, because of their complexity. From the molecular standpoint, a ganglioside presents one aspect that is exposed to the external face of a cell or a membrane, and a lipid soluble hydrophobic tail. The exposed carbohydrate moiety is normally the portion that functions as receptor, antigen and/or ligand in biological functions. Fig. 1 shows a diagram of the biosynthesis pathway of gangliosides.

Although gangliosides were initially isolated from brain and are especially abundant in neural tissues, they are widely distributed in most vertebrate tissues and fluids. Gangliosides are usually isolated from these sources by total lipid extraction and solvent partition. Total gangliosides are usually quantified as lipid bound sialic acid (LBSA) by colorimetric determination. To know the actual concentration of gangliosides in these tissues or fluids, it is necessary either to know the distribution of individual gangliosides and their molecular weight to get it from the LBSA content, or to use alternative techniques such as high performance liquid chromatography or mass spectroscopy and nuclear magnetic resonance combined with proper standards.

From a nutritional point of view the presence of gangliosides in milk, as well as the changes in content and individual profile that occur during lactation, could have special relevance.

\section{Milk gangliosides}

Milk gangliosides are almost exclusively associated with the membrane fraction of the fat globule, which is derived mainly from the apical plasma membrane of the apocrine secretory cells in the lactating mammary gland ${ }^{3}$. Milk gangliosides were initially studied in bovine milk ${ }^{4}, \mathrm{GD}_{3}$ being the major ganglioside and $\mathrm{GM}_{3}$ the next most abundant. Other gangliosides amounted to no more than $20 \%$ of the total ganglioside content. Results from several studies carried out on milk from cows, goats and ewes have recently been reported describing changes between species and during lactation, as well as seasonal variations in the concentration of gangliosides $^{5-8}$. Changes in ceramide moiety in bovine milk gangliosides with stage of lactation has also been observed 9 . 


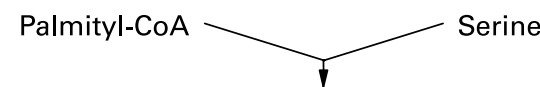

CoASH + ketodihydrosphingosine

$\mathrm{NADPH}+\mathrm{H}_{+}$

$\mathrm{NADP}+$

Dihydrosophingosine

(Sphinganine)

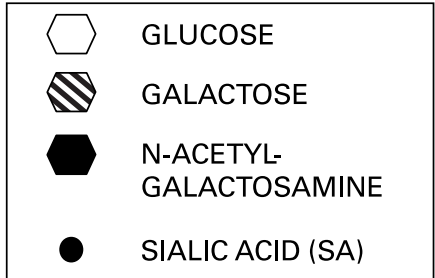

Acil-CoA $\longrightarrow$



UDP-Gal
UDP

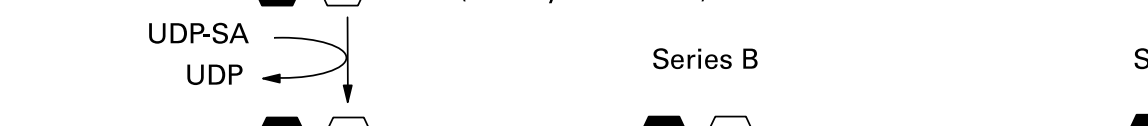

CER (glucosil-ceramida)

M4

Series A

- CER (Iactosyl-ceramide)

Series C
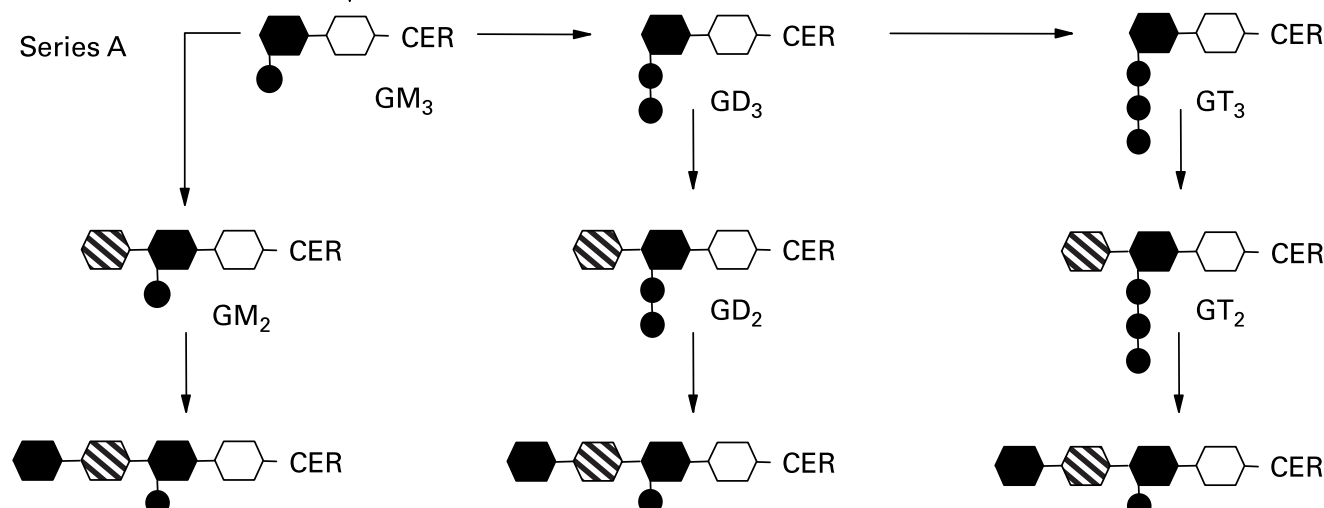

$\mathrm{GM}_{1}$

$\downarrow$


Fig. 1. Biosynthetic pathways for gangliosides. (a) Biosynthesis of lactosylceramide. (b) Biosynthesis of gangliosides from lactosylceramide. Taken from Ref. 20.

The content and distribution of gangliosides in human milk have been reported by several authors ${ }^{10-14}$. The last three studies investigated samples from different periods of lactation, and, in addition, observed a selective change in the relative concentrations of $\mathrm{GM}_{3}$ and $\mathrm{GD}_{3}$ between colostrum (days 1-5) and mature milk. The most abundant ganglioside in human milk at the beginning of lactation was $\mathrm{GD}_{3}$, while at the end of this period $\mathrm{GM}_{3}$ was the major ganglioside. A major finding in the study from our group ${ }^{13}$ was the detection of previously unreported highly polar gangliosides in human milk. Because of their high polarity they might be polysialogangliosides or complex gangliosides with branched oligosaccharide chains. 
In a different study, four gangliosides, in addition to GD3 and GM3, have been detected and assumed to tentatively be gangliosides of the c-series ${ }^{14}$.

Differences in the relative concentration of individual gangliosides in human milk from mothers delivering preterm and term infants have been reported ${ }^{15}$. In addition, changes in fatty acid composition of human milk gangliosides through lactation have been described ${ }^{16}$ and differences in fatty acid composition between bovine and human milk gangliosides have been detailed ${ }^{17}$. These studies are of interest because the lipid component of gangliosides is frequently neglected in structural characterisation studies.

Identifying specific gangliosides in complex mixtures is laborious, and requires well established validated assays or well characterised reagents, such as monoclonal antibodies or lectins. Usually, ganglioside content is estimated by means of lipid bound sialic acid (LBSA) as a surrogate parameter. For this reason it is relevant to use studies that actually measure individual ganglioside profiles, in addition to LBSA measurements, when estimating the actual concentration of gangliosides in a particular source, fluid or tissue ${ }^{12-14}$. From these studies, if the percentage of each individual ganglioside, as well as its molecular weight, are known, it is possible to calculate the actual concentration of $\mathrm{GD}_{3}$ plus $\mathrm{GM}_{3}$, the two main individual gangliosides, in human milk. Table 1 shows the average content of total gangliosides and of $\mathrm{GD}_{3}$ plus $\mathrm{GM}_{3}$, expressed as LBSA and actual concentration, for different periods of lactation, and for each one of the studies referred to above. The average value of $\mathrm{GD}_{3}$ plus $\mathrm{GM}_{3}$ in human milk, weighted according to the number of samples, would be of $13.2 \mathrm{mg} / \mathrm{L}$ considering colostrum, transitional and mature human milk, or $11.1 \mathrm{mg} / \mathrm{L}$, when only mature human milk is considered ${ }^{12-14}$.

Several authors have also studied the composition of gangliosides and other sialoglycoconjugates in infant formulas ${ }^{18,19}$. These studies have confirmed that both the pattern and content of gangliosides in human milk and infant formulas differed markedly - GD3 is the main species detected in infant formulas and their total ganglioside content is significantly lower than that of human milk.

\section{The role of dietary gangliosides on immunity and the prevention of infection}

The role of gangliosides in human milk continues to be a subject of research and discussion in the fields of paediatric nutrition and glycobiology. As described above, the distribution profile of milk gangliosides selectively changes during lactation, suggesting that gangliosides may participate in physiological processes that take place in the early development of infants $^{20,21}$.

Most of the functions in which gangliosides are implicated involve cell-cell recognition during normal differentiation or tissue targeting. It is also well known that gangliosides can be "kidnapped" by pathogenic agents and toxins that use them as unintended receptors, and that this is the main mechanism by which gangliosides can prevent infection. Human-milk gangliosides have been involved in the inhibition of Escherichia coli and Vibrio cholerae enterotoxins ${ }^{22}$. This inhibitory action was later attributed to the monosialoganglioside $\mathrm{GM}_{1}$, which has been identified as the unintended mammalian cell surface




receptor for these enterotoxins ${ }^{23}$. More recently, the free milk oligosaccharide sialyllactose, which is the oligosaccharide present in $\mathrm{GM}_{1}$, has been identified as an inhibitor of cholera toxin adhesion to target tissues ${ }^{24} \cdot \mathrm{GM}_{1}$ is found in human milk in very low concentrations, and immunological methods are necessary to detect it on high performance thin layer chromatography plates $^{23}$.

Several studies ${ }^{25-27}$ support the notion that cell surface gangliosides function as "unintended" target receptors for bacterial adhesion in specific tissues; dietary gangliosides are putative decoys that interfere with pathogenic binding. Such compounds could modify the intestinal microflora in the neonate and reduce the infectious capacity of pathogenic bacteria. Other findings also describe that sialylated compounds have growth-promoting effects on bifidobacteria and lactobacilli $^{28,29}$.

Only one clinical study has been published to date testing the role of dietary gangliosides in humans ${ }^{30}$. In this study, the performance of a formula containing gangliosides was compared to that of a control formula. To assess their impact on faecal flora, gangliosides were fed to pre-term infants, at a concentration of $1.43 \mathrm{mg} / 100 \mathrm{kcal}$, which was close to that detected in human milk by the same authors ${ }^{13}$. The molecular identity of the gangliosides to those of human milk was not the issue since, as discussed above, the most important consideration of glycoconjugates as pathogenic adhesion inhibitors is poly-valence. However, we sought to replicate human milk in terms of total ganglioside content as opposed to mimicking the average of its molecular composition and its effect on intestinal microflora. The particular impact of gangliosides on E. coli and on Bifidobacteria is shown in Fig. 2. The faecal E. coli counts in preterm infants fed the ganglioside-supplemented formula was lower than that observed in infants fed the standard formula for the first month of life. Furthermore, dietary gangliosides were also able to increase Bifidobacteria counts in faeces, which supports a prebiotic role for these glycolipids. It is difficult to pinpoint the structural features of gangliosides responsible for specific biological effects; however, it is important to keep in mind that



Fig. 2. Logarithmic microbial counts in dry faeces of preterm newborn infants fed on milk formula (MF) and milk formula supplemented with gangliosides, at $1.43 \mathrm{mg} / 100 \mathrm{kcal}(\mathrm{GMF})$. Results are means \pm SEM. Twenty samples were analised for each feeding group. Samples were collected at 3, 7 and 30 days of life. Kruskal-Wallis and Friedman nonparametric tests were used to determine the effects of diet and postnatal age as sources of variation. ${ }^{\star} P<0.01$ (versus MF group); $\dagger P<0.001$ (versus MF group); $¥ P<0.05$. Data are taken from Ref. 30. their carbohydrate portions either resemble or are identical to those of free oligosaccharides with proven prebiotic capabilities.

Another notable aspect is the ability of gangliosides to modulate the development or behaviour of cells of the immune system. Since some studies suggest that gangliosides could be involved in the activation of $\mathrm{T}$ cells ${ }^{31}$ and in the differentiation of different lymphocyte subpopulations ${ }^{32,33}$, human milk gangliosides or gangliosides added to infant formulas might substantially contribute to the processes of proliferation, activation and differentiation of immune cells, especially of those from the intestine, in the neonate. Our group reported some findings related to the effect of dietary gangliosides on intestinal immunity in mice at weaning. Animals fed with gangliosides showed an earlier development of cytokine-secreting cells, and a higher number of Th1 and Th2 cytokine-secreting lymphocytes in lamina propria and Peyer's patch lymphocytes after 4 weeks of feeding ${ }^{34}$. Another important finding was that dietary gangliosides increase the number of intestinal IgA-secreting cells ${ }^{35}$ and the luminal content of secretory $\operatorname{IgA}$ in weanling mice ${ }^{36}$. According to these results, dietary gangliosides positively modulate the production and secretion of IgA at intestinal level, which constitutes the main mechanism of defense against microorganisms entering through the gastrointestinal tract. Fig. 3 shows the effect of dietary gangliosides on the luminal content of intestinal IgA in mice at weaning. Ganglioside influences on in vitro intestinal lymphocyte proliferation were also reported ${ }^{37}$. Gangliosides elicited differential effects on intestinal lymphocyte proliferation depending on the presence and concentration of specific structures, suggesting that dietary gangliosides may influence the development of intestinal immunity by stimulating or inhibiting proliferative or inhibitory responses in intestinal lymphocytes during early infancy.

On the other hand, a recent publication describes the role of milk gangliosides, especially $\mathrm{GD}_{3}$ at early lactation (that is colostrum and transitional milk), in modulating immunity ${ }^{38}$. This publication describes how $\mathrm{GM}_{3}$ and $\mathrm{GD}_{3}$ differentially inhibit dendritic cell maturation and effector functionalities, reflecting

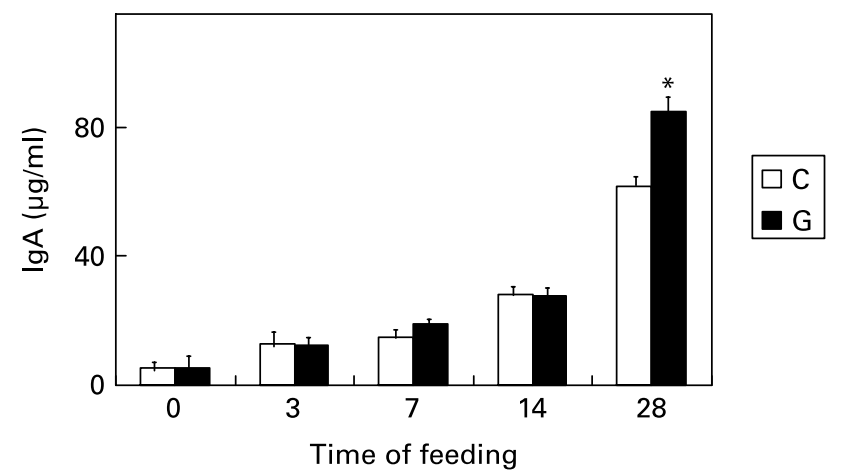

Fig. 3. Concentration of $\lg A$, normalised by intestine length and mouse weight, at the intestinal lumen of mice fed on a control diet (C) or the same diet supplemented with gangliosides, at $47 \mathrm{mg} / \mathrm{kg}(\mathrm{G})$. Samples were collected at $3,7,14$ and 28 days of feeding. Results are means \pm SEM. Two-way ANOVA and Bonferroni test were used to determine the effects of diet and time of feeding as sources of variation ${ }^{\star} P<0.05$. Data are taken from Ref. 36. 
the same compositional changes found in human milk. It is important to keep in mind that developing and modulating immunity includes not only promoting the defense against external aggression (for example infection), but also the promotion of adequate tolerance against non-aggressive antigens, which is also equally important in early infancy, and dendritic cells have a prominent role in the regulation of this process. In summary, this publication concludes that because $\mathrm{GD}_{3}$ inhibits dendritic cell functionalities overall more than $\mathrm{GM}_{3}$, we may conclude that the immune modulating effect of the ganglioside fraction of breast milk might be more prominent in the commencement of lactation during which the milk contains the most $\mathrm{GD}_{3}$.

A study has also suggested a role of gangliosides in the prevention of infection by parasites ${ }^{39}$. Results from this study showed that dietary gangliosides had a protective effect against Giardia muris infection in vivo and affected the survival of Giardia lamblia trophozoites in vitro. Several recent reports from the same group describing the effects of dietary gangliosides on neonatal rats suggest yet another role for gangliosides. One of these reports ${ }^{40}$ shows that dietary gangliosides are absorbed in the small intestine and transported to different sites, altering ganglioside levels in the intestinal mucosa, plasma and brain, and thus possibly having the potential to change developing enterocyte function. Another report ${ }^{41}$ describes that dietary gangliosides enhance in vitro lipid uptake in weanling rats, probably by a modification in the physical properties of the brush border membrane, and dietary gangliosides have been noted to increase the content and composition of phospholipids containing PUFA in the weanling rat intestine ${ }^{42}$. These recent reports suggest that dietary gangliosides might have an effect on developing enterocyte function, but it is not known if they are also related to modulation of intestinal immunity and prevention of infection.

In conclusion, dietary gangliosides may have an important role during early infancy in modifying intestinal microflora and promoting the development of intestinal immunity and oral tolerance in the neonate, and as a consequence preventing infections during early infancy. However, further studies are required to clarify the mechanisms involved in these actions and the relevance of this finding to clinical outcomes in neonates.

\section{Conflict of interest statement}

$\mathrm{RR}$ is an employee from Abbott Laboratories. Part of the work described in this article has been funded by this company.

\section{References}

1. Wiegandt H (1982) The gangliosides. In Advances in Neurochemistry, vol. 4, pp. 149-223 [BW Agranof and MH Aprison, editors]. New York: Plenum Press.

2. Svennerholm L (1963) Chromatographic separation of human brain gangliosides. J Neurochem 10, 613-623.

3. Keenan TW (1974) Composition and synthesis of gangliosides in mammary gland and milk of the bovine. Biochim Biophys Acta 337, 255-270.

4. Bushway AA \& Keenan TW (1978) Composition and synthesis of three higher ganglioside analogs in bovine mammary tissue. Lipids 13, 59-65.

5. Puente R, García-Pardo LA \& Hueso P (1992) Gangliosides in bovine milk. Changes in content and distribution of individual ganglioside levels during lactation. Biol Chem Hoppe-Seyler 373, 283-288.
6. Puente R, García-Pardo LA, Rueda R, Gil A \& Hueso P (1994) Changes in ganglioside and sialic acid contents of goat milk during lactation. J Dairy Sci 77, 39-44.

7. Puente R, García-Pardo LA, Rueda R, Gil A \& Hueso P (1995) Ewes' milk: changes in the contents of ganglioside and sialic acid during lactation. $J$ Dairy Res 62, 651-654.

8. Puente R, García-Pardo LA, Rueda R, Gil A \& Hueso P (1996) Seasonal variations in the concentration of gangliosides and sialic acids in milk from different mammalian species. Int Dairy J 6, 315-322.

9. Martín MJ, Martín-Sosa S \& Hueso P (2001) Bovine milk gangliosides: changes in ceramide moiety with stage of lactation. Lipids 36, 291-298.

10. Laegreid A, Otnaess ABK \& Fuglesang J (1986) Human and bovine milk: comparison of ganglioside composition and enterotoxin-inhibitory activity. Pediatr Res 20, 416-421.

11. Laegreid A \& Otnaess ABK (1986) Purification of human milk gangliosides by silica gel chromatography and analysis of trifluoroacetate derivatives by gas chromatography. J Chromatogr 377, 59-67.

12. Takamizawa K, Iwamori M, Mutai M \& Nagai Y (1986) Selective changes in ganglioside of human milk during lactation: a molecular indicator for the period of lactation. Biochim Biophys Acta 879, 73-77.

13. Rueda R, Puente R, Hueso P, Maldonado J \& Gil A (1995) New data on content and distribution of gangliosides in human milk. Biol Chem Hoppe-Seyler 376, 723-727.

14. Pan XL \& Izumi T (1999) Chronological changes in the ganglioside composition of human milk during lactation. Early Hum Dev 55, 1-8.

15. Rueda R, García-Salmerón JL, Maldonado J \& Gil A (1996) Changes during lactation in ganglioside distribution in human milk from mothers delivering preterm and term infants. Biol Chem 377, 599-601.

16. Martin-Sosa S, Martin MJ, Castro MD, Cabezas JA \& Hueso P (2004) Lactational changes in the fatty acid composition of human milk gangliosides. Lipids 39, 111-116.

17. Bode L, Beermann C, Mank M, Kohn G \& Boehm G (2004) Human and bovine milk gangliosides differ in their fatty acid composition. J Nutr 134, 3016-3020.

18. Sánchez-Díaz A, Ruano MJ, Lorente F \& Hueso P (1997) A critical análisis of total sialic acid and sialoglycoconjugate contents of bovine milk-based infant formulas. J Pediatr Gastroenterol Nutr 24, 405-410.

19. Pan XL \& Izumi T (2000) Variation of the ganglioside compositions of human milk, cow's milk and infant formulas. Early Hum Dev 57, 25-31.

20. Rueda R \& Gil A (1998) Role of gangliosides in infant nutrition. In Lipids in Infant Nutrition, pp. 213-234 [Y-S Huang and AJ Sinclair, editors]. Champaign, IL: AOCS Press.

21. Rueda R, Maldonado J, Narbona E \& Gil A (1998) Neonatal dietary gangliosides. Early Hum Dev 53, S135-S147.

22. Otnaess ABK, Laegreid A \& Ertresrag K (1983) Inhibition of enterotoxin from Escherichia coli and Vibrio cholerae by gangliosides from human milk. Infect Immun 40, 563-569.

23. Laegreid A \& Otnaess ABK (1987) Trace amounts of ganglioside GM1 in human milk inhibits enterotoxin from Vibrio cholerae and Escherichia coli. Life Sci 40, 55-62.

24. Idota T, Kawakami H, Murakami Y \& Sugawara M (1995) Inhibition of cholera toxin by human milk fractions and sialyllactose. Biosci Biotech Biochem 59, 417-419.

25. Teneberg S, Willemsen P, de Graaf FK \& Karlsson KA (1993) Calf small intestine receptors for K99 fimbriated enterotoxigenic Escherichia coli. FEMS Microbiol Lett 109, 107-112.

26. Yuyama Y, Yoshimatsu K, Ono E, Saito M \& Naiki M (1993) Postnatal change of pig intestinal ganglioside bound by Escherichia coli with K99 fimbriae. J Biochem 113, 488-492. 
27. Idota $\mathrm{T} \&$ \& Kawakami H (1995) Inhibitory effects of milk gangliosides on the adhesion of Escherichia coli to human intestinal carcinoma cells. Biosci Biotech Biochem 59, 69-72.

28. Idota T, Kawakami H \& Nakajima I (1994) Growth-promoting effects of N-Acetylneuraminic acid-containing substances on bifidobacteria. Biosci Biotech Biochem 58, 1720-1722.

29. Nakano T, Sugawara M \& Kawakami H (2001) Sialic acid in human milk: composition and functions. Acta Paediatr Taiwan 42, 11-17.

30. Rueda R, Sabatel JL, Maldonado J, Molina JA \& Gil A (1998b) Addition of gangliosides to an adapted milk formula modifies the levels of fecal Escherichia coli in preterm newborn infants. J Pediatr 133, 90-94.

31. Yuasa H, Scheinberg DA \& Houghton AN (1990) Gangliosides of T Lymphocytes: evidence for a role in T-Cell activation. Tissue Antigens 36, 47-56.

32. Ebel F, Scmitt E, Peter-Katalinic J, Kniep B \& Mühlradt PF (1992) Gangliosides: differentiation markers for murine $\mathrm{T}$ helper lymphocyte subpopulations TH1 and TH2. Biochemistry 31, 12190-12197.

33. Taga S, Tetaud C, Mangeney M, Tursz T \& Wiels J (1995) Sequential changes in glycolipid expression during human B cell differentiation: enzymatic basis. Biochim Biophys Acta 1254, 56-65.

34. Vázquez E, Gil A \& Rueda R (2001) Dietary gangliosides positively modulate the percentages of Th1 and Th2 lymphocyte subsets in small intestine of mice at weaning. BioFactors 15, 1-9.

35. Vázquez E, Gil A, García-Olivares E \& Rueda R (1999) Dietary gangliosides increase the number of intestinal IgA-secreting cells in weanling mice. Immunol Letters 69, 44-47.
36. Vázquez E, Gil A \& Rueda R (2000) Dietary gangliosides increase the number of intestinal IgA-secreting cells and the luminal content of secretory $\operatorname{IgA}$ in weanling mice. $J$ Pediatr Gastroenterol Nutr 31, Suppl. 2, S133.

37. Vázquez E, Gil A \& Rueda R (2002) Low concentration of gangliosides strongly stimulate DNA synthesis in cultured resting intestinal lymphocytes from young mice. In $11^{\text {th }}$ International Congress of Mucosal Immunology, p. 1301. USA: Orlando.

38. Bronnum H, Seested T, Hellgren LI, Brix S \& Frokiaer H (2005) Milk-derived $\mathrm{GM}_{3}$ and $\mathrm{GD}_{3}$ differentially inhibit dendritic cell maturation and effector functionalities. Scand J Immunol 61, 551-557.

39. Suh M, Belosevic M \& Clandinin MT (2004) Dietary lipids containing gangliosides reduce Giardia muris infection in vivo and survival of Giardia lamblia trophozoites in vitro. Parasitology 128, 595-602.

40. Park EJ, Suh M, Ramanujam K, Steiner K, Begg D \& Clandinin MT (2005) Diet-induced changes in membrane gangliosides in rat intestinal mucosa, plasma and brain. J Pediatr Gastroenterol Nutr 40, 487-495.

41. Birecki CJ, Drozdowski LA, Suh M, Park EJ, Clandinin MT \& Thomson AB (2006) Dietary gangliosides enhance in vitro lipid uptake in weanling rats. J Pediatr Gastroenterol Nutr 42, 59-65.

42. Park EJ, Suh M, Thomson AB, Ramanujam KS \& Clandinin MT (2006) Dietary gangliosides increase the content and percentage of ether phospholipids containing 20:4n-6 and 22:6n-3 in weanling rat intestine. $J$ Nutr Biochem 17, 337-344. 